MATHEMATICS OF COMPUTATION

S 0025-5718(99)01098-4

Article electronically published on April 7, 1999

\title{
A PARALLEL METHOD \\ FOR TIME-DISCRETIZATION OF PARABOLIC PROBLEMS BASED ON CONTOUR INTEGRAL REPRESENTATION AND QUADRATURE
}

\author{
DONGWOO SHEEN, IAN H. SLOAN, AND VIDAR THOMÉE
}

\begin{abstract}
We treat the time discretization of an initial-value problem for a homogeneous abstract parabolic equation by first using a representation of the solution as an integral along the boundary of a sector in the right half of the complex plane, then transforming this into a real integral on the finite interval $[0,1]$, and finally applying a standard quadrature formula to this integral. The method requires the solution of a finite set of elliptic problems with complex coefficients, which are independent and may therefore be done in parallel. The method is combined with spatial discretization by finite elements.
\end{abstract}

\section{INTRODUCTION}

We consider the initial-value problem

$$
u_{t}+A u=0, \quad \text { for } t>0, \quad \text { with } u(0)=u_{0},
$$

where $A$ is a symmetric, positive definite operator with a compact inverse, defined on a dense subset $D$ of a Hilbert space $H$, with inner product $(\cdot, \cdot)$. The solution may be written in the form (cf. [8], Theorem 1.7.7)

$$
u(t)=\frac{1}{2 \pi \mathrm{i}} \int_{\Gamma} e^{-z t} R(z ; A) u_{0} d z, \quad \text { for } t>0,
$$

where $R(z ; A)=(A-z I)^{-1}$ is the negative of the resolvent of $A$, and $\Gamma$ is a conveniently chosen path in the right half plane. With the minimal eigenvalue of $A$ bounded below by $\lambda_{0}>0$, and with $0 \leq \gamma<\lambda_{0}$, we shall use $\Gamma=\Gamma_{\gamma}=\{z=$ $\gamma+\sigma \pm \mathrm{i} \sigma ; \sigma \geq 0\}$, with $\operatorname{Im} z$ increasing from $-\infty$ to $\infty$. This representation may be obtained by applying the Laplace transform, defined by

$$
w(z)=\hat{u}(z)=\int_{0}^{\infty} e^{z t} u(t) d t, \quad \text { for } \operatorname{Re} z \leq \gamma,
$$

to the initial value problem (1.1). In this way we derive

$$
A w-z w=u_{0}, \quad \text { i.e., } \quad w(z)=R(z ; A) u_{0}, \quad \text { for } \operatorname{Re} z \leq \gamma,
$$

Received by the editor March 26, 1998.

1991 Mathematics Subject Classification. Primary 65M12, 65M15, 65M99.

This work was partially supported by the Australian Research Council and the Korea Science \& Engineering Foundation through the Global Analysis Research Center at Seoul National University. 
and then retrieve $u(t)$ by the inverse of the Laplace transform (1.3) taken along $\{z ; \operatorname{Re} z=\gamma\}$, subsequently deforming this path to $\Gamma_{\gamma}$. This is possible since $w(z)=R(z ; A) u_{0}$ exists for $z \in \mathbb{C} \backslash\left[\lambda_{0}, \infty\right)$, and since $\|R(z ; A)\| \leq C(|z|+1)^{-1}$ for $z$ bounded away from $\left[\lambda_{0}, \infty\right)$. Since $A$ is self-adjoint the representation (1.2) may also be established by deforming $\Gamma$ into a union of small circles around the eigenvalues of $A$, in which case (1.2) reduces to the eigenfunction expansion of $u(t)$.

The contour integral representation (1.2) of $u(t)$ may be written as

$$
u(t)=\int_{0}^{\infty} e^{-(\gamma+\sigma) t} g(t ; \sigma) d \sigma
$$

where

$$
g(t ; \sigma)=\frac{1}{2 \pi \mathrm{i}}\left(\nu e^{-\mathrm{i} \sigma t} w(\gamma+\nu \sigma)-\bar{\nu} e^{\mathrm{i} \sigma t} w(\gamma+\bar{\nu} \sigma)\right), \quad \text { with } \nu=1+\mathrm{i} .
$$

In our applications $w$ will be a complex-valued function, satisfying $\overline{w(z)}=w(\bar{z})$ because of $(1.4)$, in which case $g(t ; \sigma)$ is real, and

$$
g(t ; \sigma)=\frac{1}{\pi} \operatorname{Im}\left(\nu e^{-\mathrm{i} \sigma t} w(\gamma+\nu \sigma)\right) .
$$

Our approach to the approximate solution of (1.1) is to apply a quadrature scheme to (1.5). We shall later also apply our method to the discretization in time of an initial value problem of the form (1.1) which has been obtained from an initial boundary value problem for a parabolic partial differential equation by first discretizing in the space variables by finite elements.

We consider quadrature approximations to the integral in (1.5) of the form

$$
U(t)=\sum_{j} \omega_{j}(t) g\left(t ; \sigma_{j}\right), \quad \text { for } t>0
$$

with non-negative quadrature points $\sigma_{j}$ and positive quadrature weights $\omega_{j}(t)$. The construction of such a quadrature rule will be accomplished by first changing the variable in (1.5) to $y=e^{-\alpha \sigma t}$, where $\alpha$ is a positive number to be specified later, obtaining

$$
u(t)=\frac{e^{-\gamma t}}{\alpha t} \int_{0}^{1} y^{-1+1 / \alpha} g(t ; \sigma(y)) d y, \quad \text { with } \sigma(y)=\frac{1}{\alpha t} \log \frac{1}{y},
$$

and then applying a standard quadrature rule. For simplicity, our first approximation uses the composite trapezoidal rule based on a uniform partition of $[0,1]$, $0=y_{0}<y_{1}<\cdots<y_{N}=1$, where $y_{j}=j / N, j=0, \cdots, N$. We recall that for the integral If $=\int_{0}^{1} f(y) d y$ the composite trapezoidal rule is

$$
T_{N} f=\frac{1}{2 N}\left(f\left(y_{0}\right)+2 f\left(y_{1}\right)+2 f\left(y_{2}\right)+\cdots+2 f\left(y_{N-1}\right)+f\left(y_{N}\right)\right)
$$

and that the quadrature error satisfies (see [1], p. 220)

$$
\left|T_{N} f-I f\right| \leq \frac{1}{8 N^{2}} \int_{0}^{1}\left|f^{\prime \prime}(y)\right| d y .
$$

Applying this with $f(y)=(\alpha t)^{-1} y^{-1+1 / \alpha} g(t ; \sigma(y))$, we get a quadrature rule of the form (1.8), with

$$
\sigma_{j}=\frac{1}{\alpha t} \log \frac{1}{y_{j}}=\frac{1}{\alpha t} \log \frac{N}{j}, \quad j=0, \ldots, N,
$$


and the corresponding weights (except the first and the last, which have an additional factor of $\frac{1}{2}$ ) are

$$
\omega_{j}(t)=\frac{e^{-\gamma t}}{\alpha t N} y_{j}^{-1+1 / \alpha}=\frac{e^{-\gamma t}}{\alpha t N}\left(\frac{j}{N}\right)^{-1+1 / \alpha} .
$$

The positive number $\alpha$ is then chosen so as to make the error bound in (1.11) small. It turns out, as we shall see below, that

$$
\left\|f^{\prime \prime}(y)\right\| \leq C(\alpha, t) y^{-3+1 / \alpha}\left\|u_{0}\right\|, \quad \text { for } y \in[0,1],
$$

and hence the integral in the vector version of (1.11) is finite for $\alpha<1 / 2$, so that the error in that situation is of optimal order $O\left(N^{-2}\right)$.

Assume now that the solution is sought for $t>\tau$, where $\tau$ is a positive number chosen by the user. For the method to be efficient it is crucial that the same set of points $\sigma_{j}$ be used for a whole range of $t$ values. Since the $\sigma_{j}$ depend on $\alpha$ and $t$ only through the product $\alpha t$, this product should thus be held constant in a time interval $[\tau, T]$, implying that $\alpha$ must vary with $t$. In view of the above it is natural to base the choice of the $\sigma_{j}$ on $\alpha t=\frac{1}{2} \tau$, or $\alpha=\frac{1}{2} \tau / t$, because $t>\tau$ is then equivalent to $\alpha<1 / 2$.

Having thus fixed $\tau$ and chosen $\alpha=\frac{1}{2} \tau / t$, thereby securing $O\left(N^{-2}\right)$ convergence for each $t>\tau$, we may still ask for the properties of the method for $t \leq \tau$, or for $\alpha \geq 1 / 2$. Since $f\left(y_{0}\right)=f(0)=0$ if $\alpha<1$ because $g(t ; \sigma)$ is bounded, the quadrature point at $y=0$ (corresponding to $\sigma=\infty$ ) does not contribute to the quadrature sum for $\alpha<1$. If $\alpha \geq 1$, we define the quadrature sum so as to omit the $y=0$ term; that is, we "ignore the singularity" (see [1], Section 2.12.7) in the situation in which there is an endpoint singularity. Thus our quadrature sums always run only from $j=1$ to $j=N$. With this understanding we shall be able to show error estimates for $0<t \leq \tau$ which are of lower order than $O\left(N^{-2}\right)$; the order is $O\left(N^{-2 t / \tau}\right)$ for $0<t<\tau$, with an additional factor $\log \log N$ when $t=\tau$. A full statement is in Theorem 2.2.

Thus, the definition of our method may be summarized as follows: We first choose $\gamma \in\left[0, \lambda_{0}\right)$ and the threshold $\tau>0$, then for $t$ and $\alpha$ satisfying $\alpha t=\frac{1}{2} \tau$ we determine $\sigma_{j}=2 \tau^{-1} \log (N / j), j=1, \ldots, N$, then solve the $N$ complex-valued elliptic problems (1.4) with $z=\gamma+\nu \sigma_{j}, \nu=1+\mathrm{i}$, and finally form $g\left(t ; \sigma_{j}\right)$ and $U(t)=U_{\tau}(t)$ from (1.8), (1.6), and (1.13). In the fully discrete case the elliptic problems (1.4) are solved approximately by the finite element method.

Instead of the trapezoidal rule we can apply the composite Simpson rule (provided $N$ is even),

$$
S_{N} f=\frac{1}{3 N}\left(f\left(y_{0}\right)+4 f\left(y_{1}\right)+2 f\left(y_{2}\right)+4 f\left(y_{3}\right)+\cdots+4 f\left(y_{N-1}\right)+f\left(y_{N}\right)\right)
$$

with the error estimate

$$
\left|S_{N} f-I f\right| \leq \frac{C}{N^{4}} \int_{0}^{1}\left|f^{(i v)}(y)\right| d y .
$$

In this case it turns out, as we shall see, that the full $O\left(N^{-4}\right)$ convergence order is obtained only for $\alpha<\frac{1}{4}$, and it is then appropriate to choose $\alpha$ so that $\alpha t=\frac{1}{4} \tau$, to secure again the full order of convergence for $t>\tau$. Once more convergence rates of lower orders may be shown for $t \leq \tau$. The full Simpson's rule result is stated as Theorem 2.3. 
We now turn to discretization in both space and time of an initial boundary value problem for a parabolic partial differential equation. For simplicity we consider the case of the heat equation, viz.

$$
\begin{gathered}
u_{t}-\Delta u=0 \quad \text { in } \Omega, \quad \text { with } u=0 \quad \text { on } \partial \Omega, \text { for } t>0, \\
u(\cdot, 0)=u_{0} \quad \text { in } \Omega,
\end{gathered}
$$

where $\Omega \subset R^{d}$ is a domain with smooth boundary $\partial \Omega$. We thus choose the Hilbert space $H$ as $L_{2}(\Omega)$ with $(v, w)=\int_{\Omega} v(x) w(x) d x$. Here $A=-\Delta$, the Laplacian, which is defined in $D(A)=H^{2}(\Omega) \cap H_{0}^{1}(\Omega)$. Setting

$$
A(v, z)=\int_{\Omega} \nabla v(x) \cdot \nabla z(x) d x
$$

and letting $V_{h}$ denote piecewise linear finite element subspaces with standard properties, the finite element approximation $w_{h}(z) \in V_{h}$ of the solution $w$ of (1.4) satisfies

$$
A\left(w_{h}, \chi\right)-z\left(w_{h}, \chi\right)=\left(u_{0}, \chi\right), \quad \forall \chi \in V_{h}, \quad \text { for } z \notin\left[\lambda_{0}, \infty\right) .
$$

The semidiscrete approximation $u_{h}(t) \in V_{h}$ to (1.1) is defined by

$$
\left(u_{h, t}, \chi\right)+A\left(u_{h}, \chi\right)=0, \quad \forall \chi \in V_{h}, \quad \text { for } t>0, \quad \text { with } u_{h}(0)=P_{h} u_{0},
$$

where $P_{h}$ denotes the orthogonal projection in $L_{2}(\Omega)$ onto $V_{h}$. Defining the discrete analogue $A_{h}: V_{h} \rightarrow V_{h}$ of $A=-\Delta$ by

$$
\left(A_{h} \psi, \chi\right)=A(\psi, \chi), \quad \forall \psi, \chi \in V_{h},
$$

we see that (1.18) is of the form (1.1) with $A$ replaced by $A_{h}$. Similarly, (1.17) is of the form (1.4). It may be proved, analogously to (1.2) and (1.5), that

$$
u_{h}(t)=\frac{1}{2 \pi \mathrm{i}} \int_{\Gamma} e^{-z t} w_{h}(z) d z=\int_{0}^{\infty} e^{-(\gamma+\sigma) t} g_{h}(t ; \sigma) d \sigma,
$$

where (cf. (1.7)) $g_{h}(t ; \sigma)=\operatorname{Im}\left(\nu e^{-\mathrm{i} \sigma t} w_{h}(\gamma+\nu \sigma)\right) / \pi$. Our fully discrete approximation $U_{h}(t)$ is then obtained by application of the quadrature approximation (1.8) to this integral, so that

$$
U_{h}(t)=\sum_{j=1}^{N} \omega_{j}(t) g_{h}\left(t ; \sigma_{j}\right) .
$$

It will follow from our main result that in the trapezoidal rule case $\left\|U_{h}(t)-u_{h}(t)\right\|=$ $O\left(N^{-2}\right)$ for $t>\tau$, uniformly in $h$, with lower orders of convergence for $t \leq \tau$. Together with the known estimate $\left\|u_{h}(t)-u(t)\right\|=O\left(h^{2}\right)$ for $t>0$, this will give a complete error estimate of order $O\left(N^{-2}+h^{2}\right)$ for the fully discrete problem in this case (see Section 3). Similarly, in the Simpson rule case the complete error estimate is of order $O\left(N^{-4}+h^{2}\right)$.

The error bounds to be shown later limit the range of $t$-values that can effectively be covered with fixed quadrature points $\sigma_{j}$; for example, for $\gamma=0$ we shall see that restriction to an interval $\tau \leq t \leq 2 \tau$ might be appropriate, while for $\gamma>0$ a larger interval could be suitable - see Tables 1-3 and associated discussion. The error bounds will also show that this is not a method of preference for small times $t$.

We remark that our method is introduced and studied so far only for a homogeneous parabolic equation. However, the initial value problem for the inhomogeneous 
equation,

$$
u_{t}+A u=f, \quad \text { for } t>0, \quad \text { with } u(0)=u_{0},
$$

in the special case in which $f$ is independent of time, may be reduced to an initialvalue problem of the form (1.1). In fact, let $u_{\infty}$ denote the stationary solution of (1.20) defined by $A u_{\infty}=f$. Then the solution of (1.20) may be written $u(t)=$ $u_{\infty}+v(t)$, where

$$
v_{t}+A v=0, \quad \text { for } t>0, \quad \text { with } v(0)=u_{0}-u_{\infty},
$$

and our method may be applied to determine $v(t)$.

In the finite element application to this problem it is natural to take as discrete initial values $v_{h}(0)=P_{h} u_{0}-T_{h} f$, where $T_{h}=A_{h}^{-1} P_{h}$, with $A_{h}$ and $P_{h}$ as above. This differs from the initial value suggested by (1.18), which is $P_{h}\left(u_{0}-u_{\infty}\right)$, by $T_{h} f-P_{h} u_{\infty}=\left(T_{h}-P_{h} T\right) f$, where $T=A^{-1}$. By the stability of the solution operator for the homogeneous semidiscrete equation the contribution to the error of this difference is bounded by $C h^{2} e^{-\lambda_{0} t}\|f\|$, see, e.g., [10].

In the title we described this as a parallel method. The reason is clear from the formula (1.19). To compute $U_{h}(t)$ for a range of times $t \geq \tau$ we need to solve the finite element problems (1.17) for $z=\gamma+\nu \sigma_{j}, j=1, \ldots, N$. These problems are completely independent, and can therefore be computed on separate processors, with no need for shared memory. In contrast, the normal step-by-step time-marching methods for parabolic problems are not easily parallelizable.

Our method, like the method of eigenfunction expansion, requires that the operator $A$ be independent of time and symmetric positive definite. However, the representation (1.2) is valid for more general operators $A$ that generate analytic semigroups, and our time discretization method naturally extends to this case; we plan to return to the analysis of such problems on a later occasion.

Numerical methods for inhomogeneous parabolic and hyperbolic equations based on the use of Fourier transformation have been considered in [2], [3], [7], and [9], and provided the starting point for the present work. For other methods for parabolic equations of non-timestepping type, see, e.g., [11, Chapter 9], [5], and [6].

\section{Analysis of the QUADRATURE SChEme}

We recall that in addition to (1.2) the solution of (1.1) admits the representation

$$
u(t)=E(t ; A) u_{0}=\sum_{l=1}^{\infty} e^{-\lambda_{l} t}\left(u_{0}, \varphi_{l}\right) \varphi_{l},
$$

where $\left\{\varphi_{l}\right\}_{l=1}^{\infty}$ and $\left\{\lambda_{l}\right\}_{l=1}^{\infty}$ are a basis of orthonormal eigenfunctions and corresponding eigenvalues of $A$, and where $E(t ; A)=e^{-A t}$ is the semigroup generated by $-A$. Similarly, the approximate solution defined by (1.8) and (1.6) may be expressed as

$$
U(t)=Q(t ; A) u_{0}=\sum_{l=1}^{\infty} Q\left(t ; \lambda_{l}\right)\left(u_{0}, \varphi_{l}\right) \varphi_{l},
$$

where $Q(t ; A)$ is a rational function of $A$, viz.

$$
Q(t ; A)=\sum_{j=1}^{N} \omega_{j}(t) \frac{1}{\pi} \operatorname{Im}\left(\nu e^{-\mathrm{i} \sigma_{j} t} R\left(\gamma+\nu \sigma_{j} ; A\right)\right) ;
$$


recall that the imaginary part of a bounded linear operator $B$ on the Hilbert space $H$ is defined by $\operatorname{Im} B=\left(B-B^{*}\right) /(2 \mathrm{i})$. This makes it possible below to reduce the proofs of the stability and error estimates to the scalar case

$$
u^{\prime}+\lambda u=0, \quad \text { for } t>0, \quad \text { with } u(0)=1 .
$$

We suppose first that the time discretization is accomplished by the trapezoidal rule, and show the following stability property of our time discretization operator.

Proposition 2.1. Let $\tau>0$, and assume that the eigenvalues of $A$ are bounded below by $\lambda_{0}$, and let $0 \leq \gamma<\lambda_{0}$. Let $U(t)=Q(t ; A) u_{0}$ be the $N$-point trapezoidal rule approximation to (1.9), with $g(t ; \sigma)$ given by (1.6), and with $\alpha t=\frac{1}{2} \tau$. Then there is a constant $C$ independent of $t$ and $\tau$ such that, with $\log _{+} x=\max (\log x, 0)$,

$$
\|U(t)\|=\left\|Q(t ; A) u_{0}\right\| \leq C e^{-\gamma t}\left(\frac{1}{1+t}+\log _{+} \frac{1}{t}+\frac{1}{\tau} \frac{1}{N^{\min (1,2 t / \tau)}}\right)\left\|u_{0}\right\|, \quad t>0 .
$$

Proof. It suffices to show this for the scalar problem (2.2), with a constant $C$ independent of $\lambda \geq \lambda_{0}$. In fact, by (2.1),

$$
\left\|Q(t ; A) u_{0}\right\|=\left(\sum_{l=1}^{\infty} Q\left(t ; \lambda_{l}\right)^{2}\left(u_{0}, \varphi_{l}\right)^{2}\right)^{1 / 2} \leq \max _{l}\left|Q\left(t ; \lambda_{l}\right)\right|\left\|u_{0}\right\|,
$$

so it suffices to show that

$$
|Q(t ; \lambda)| \leq C e^{-\gamma t}\left(\frac{1}{1+t}+\log _{+} \frac{1}{t}+\frac{1}{\tau} \frac{1}{N^{\min (1,2 t / \tau)}}\right) \quad \text { for } \lambda \geq \lambda_{0},
$$

where it follows from the expression above that

$$
Q(t ; \lambda)=\sum_{j=1}^{N} \omega_{j}(t) \frac{1}{\pi} \operatorname{Im}\left(\nu e^{-\mathrm{i} \sigma_{j} t}\left(\lambda-\gamma-\nu \sigma_{j}\right)^{-1}\right) .
$$

Recalling that $\nu=1+\mathrm{i}$, we have, for $\lambda \geq \lambda_{0}>\gamma \geq 0$, with $C=C\left(\lambda_{0}-\gamma\right)$,

$$
\frac{1}{\pi}\left|\operatorname{Im}\left(\nu e^{-\mathrm{i} \sigma t}(\lambda-\gamma-\nu \sigma)^{-1}\right)\right| \leq C|\lambda-\gamma-\nu \sigma|^{-1} \leq C(\sigma+1)^{-1} .
$$

Hence

$$
|Q(t ; \lambda)| \leq C \sum_{j=1}^{N} \omega_{j}(t)\left(\sigma_{j}+1\right)^{-1} \leq C \frac{e^{-\gamma t}}{\alpha t N} \sum_{j=1}^{N} y_{j}^{-1+1 / \alpha}\left(\sigma\left(y_{j}\right)+1\right)^{-1}=C e^{-\gamma t} J_{N},
$$

where $J_{N}$ is a Riemann sum for the first integral in

$$
J=\frac{1}{\alpha t} \int_{0}^{1} y^{-1+1 / \alpha}(\sigma(y)+1)^{-1} d y=\int_{0}^{\infty} e^{-\sigma t}(\sigma+1)^{-1} d \sigma .
$$

For $\alpha \leq 1$, i.e., for $t \geq \frac{1}{2} \tau$, the function $y^{-1+1 / \alpha}(\sigma(y)+1)^{-1}$ is increasing from 0 to 1 as $y$ increases from 0 to 1 , and therefore the sum of the first $N-1$ terms of $J_{N}$ is bounded by $J$, so that

$$
J_{N} \leq J+\frac{1}{\alpha t N}=J+\frac{2}{\tau N} .
$$

The bound (2.3) is therefore proved for $2 t / \tau \geq 1$ by

$$
J=e^{t} \int_{t}^{\infty} \frac{e^{-x}}{x} d x \leq C\left\{\begin{array}{ll}
1+\log \frac{1}{t}, & t \leq 1 \\
\frac{1}{t}, & t \geq 1
\end{array}\right\} \leq C\left(\frac{1}{1+t}+\log _{+} \frac{1}{t}\right) .
$$


It remains to bound $J_{N}$ when $\alpha>1$, i.e., to prove (2.3) for $2 t / \tau<1$. We then need to handle the product of the decreasing function $y^{-1+1 / \alpha}$ and the increasing function $(\sigma(y)+1)^{-1}$. For this purpose we first show that

$$
\left(\sigma\left(y_{j}\right)+1\right)^{-1} \leq C\left(\sigma\left(y_{j-1}\right)+1\right)^{-1}, \quad \text { for } 2 \leq j \leq N-1,
$$

with $C$ independent of $j$ and $N$, from which it will follow for $2 \leq j \leq N-1$ that

$$
\begin{gathered}
N^{-1} y_{j}^{-1+1 / \alpha}\left(\sigma\left(y_{j}\right)+1\right)^{-1} \leq C N^{-1} y_{j}^{-1+1 / \alpha}\left(\sigma\left(y_{j-1}\right)+1\right)^{-1} \\
\leq C \int_{y_{j-1}}^{y_{j}} y^{-1+1 / \alpha}(\sigma(y)+1)^{-1} d y
\end{gathered}
$$

and hence, by summation of this bound over $j=2, \ldots, N-1$,

$$
J_{N} \leq C\left(J+\frac{1}{\tau N^{1 / \alpha}}+\frac{1}{\tau N}\right) \leq C\left(J+\frac{1}{\tau} \frac{1}{N^{\min (1,2 t / \tau)}}\right) .
$$

Since $\sigma(y)=2 \log (1 / y) / \tau$, the claim (2.5) is equivalent to showing the boundedness of $\left(\log \left(1 / y_{j-1}\right)+\tau / 2\right) /\left(\log \left(1 / y_{j}\right)+\tau / 2\right)$ for $\tau \geq 0,2 \leq j \leq N$, or (since this ratio takes its maximum value at $\tau=0)$ that $\varphi(y)=\log (y-k) / \log y$ is bounded in $[2 k, 1-k]$, where $k=1 / N$. But $\varphi(y) \rightarrow \infty$ when $y \rightarrow k$ and $y \rightarrow 1$. It then suffices to show that $\varphi(2 k)$ and $\varphi(1-k)$ are bounded, and that $\varphi(y)$ has only one stationary point in $(2 k, 1-k)$ (which then has to be a minimum). We have

$$
\varphi(2 k)=\frac{\log N}{\log (N / 2)} \leq \frac{\log 3}{\log (3 / 2)}, \quad \text { for } N \geq 3,
$$

and

$$
\varphi(1-k)=\frac{\log (1-2 k)}{\log (1-k)}=2+O\left(\frac{1}{N}\right), \quad \text { as } N \rightarrow \infty,
$$

so that both $\varphi(2 k)$ and $\varphi(1-k)$ are bounded for $N \geq 3$. A stationary point $y$ of $\varphi(y)$ has to satisfy $(y-k)^{-1} \log y-y^{-1} \log (y-k)=0$, or $g(y)=g(y-k)$, where $g(y)=y \log y$. But $g(0)=g(1)=0, g(y)<0$ for $y \in(0,1)$, and $g^{\prime}(y)=0$ only for $y=e^{-1}$, so that $g(y)=g(y-k)$ has exactly one solution in $(k, 1)$ for $k<1$. Thus the claim is established, and the proof is complete.

Note that the stability bound contains a term with a factor $\tau^{-1}$, and thus is not uniformly bounded for small $\tau$.

Let us remark that, with a slightly modified definition of the approximate solution $U(t)$, and with a mild regularity assumption on $u_{0}$, it is possible to show uniform boundedness of the time discrete solution as $t$ tends to 0 for fixed $\tau>0$. In fact, writing instead of (1.2) for the exact solution

$$
u(t)=\frac{1}{2 \pi \mathrm{i}} \int_{\Gamma_{\gamma}} e^{-z t}(1+z)^{-\epsilon} R(z ; A)(I+A)^{\epsilon} u_{0} d z, \quad \text { with } \epsilon>0,
$$

we may define an approximate solution $U_{\epsilon}(t)$ by

$$
U_{\epsilon}(t)=Q_{\epsilon}(t ; A)(I+A)^{\epsilon} u_{0},
$$

where

$$
Q_{\epsilon}(t ; A)=\sum_{j=1}^{N} \omega_{j}(t) \frac{1}{\pi} \operatorname{Im}\left(\nu e^{-\mathrm{i} \sigma_{j} t}\left(1+\gamma+\nu \sigma_{j}\right)^{-\epsilon} R\left(\gamma+\nu \sigma_{j} ; A\right)\right) .
$$


With this choice we first have to apply $(I+A)^{\epsilon}$ to the given initial data; for $\epsilon=1$ this simply entails application of $A$. Similarly to our earlier estimate, we find in the scalar case $A=\lambda$ that

$$
\begin{aligned}
\left|Q_{\epsilon}(t ; \lambda)\right| & \leq \frac{e^{-\gamma t}}{\alpha t N} \sum_{j=1}^{N} y_{j}^{-1+1 / \alpha}\left(\sigma_{j}+1\right)^{-1-\epsilon} \\
& \leq C e^{-\gamma t}\left(\int_{0}^{\infty} e^{-t \sigma}(\sigma+1)^{-1-\epsilon} d \sigma+\frac{1}{\tau} \frac{1}{N^{\min (1,2 t / \tau)}}\right) \\
& \leq C e^{-\gamma t}\left(\frac{1}{1+t}+\frac{1}{\tau} \frac{1}{\left.N^{\min (1,2 t / \tau)}\right)} .\right.
\end{aligned}
$$

Thus, in this case, since $(I+A)^{\epsilon} A^{-\epsilon}$ is bounded in $H$,

$$
\left\|U_{\epsilon}(t)\right\| \leq C e^{-\gamma t}\left(\frac{1}{1+t}+\frac{1}{\tau} \frac{1}{\left.N^{\min (1,2 t / \tau)}\right)}\right)\left\|A^{\epsilon} u_{0}\right\|, \quad \text { for } t \geq 0,
$$

in which there is no longer any logarithmic behavior as $t \rightarrow 0$.

We now return to the main theme and show an error estimate for the approximate solution $U(t)$ defined by the trapezoidal rule.

Theorem 2.2. Let $\tau>0$ and assume the eigenvalues of $A$ are bounded below by $\lambda_{0}$, and let $0 \leq \gamma<\lambda_{0}$. Assume that the quadrature approximation $U(t)$ is obtained by applying the trapezoidal rule (1.10) (with the $y_{0}$ term omitted) to the integral in (1.9), where $g(t ; \sigma)$ is defined by (1.6), and $\alpha$ is chosen so that $\alpha t=\tau / 2$. Then there exists $C=C\left(\lambda_{0}-\gamma\right)>0$, such that

$$
\|U(t)-u(t)\| \leq C\left\|u_{0}\right\| e^{-\gamma t} \begin{cases}\frac{1}{N^{2}}\left(\frac{1+t^{2}}{\tau^{2}(1+t-\tau)}+\frac{t^{2}}{\tau^{2}} \log _{+} \frac{1}{t-\tau}\right), & t>\tau, \\ \frac{1}{N^{2}}\left(\log \log N+\frac{1}{\tau^{2}}+\log _{+} \frac{1}{\tau}\right), \quad N \geq 3, & t=\tau, \\ \frac{1}{N^{2 t / \tau}}\left(\frac{1+\tau^{2}}{\tau^{2}}+\log _{+} \frac{1}{\tau-t}+\log _{+} \frac{1}{t}\right), & 0<t<\tau .\end{cases}
$$

Proof. Again it suffices to consider the scalar problem (2.2), since it follows in the same way as in the proof of Proposition 2.1 that

$$
\|U(t)-u(t)\| \leq \max _{l}\left|Q\left(t ; \lambda_{l}\right)-e^{-\lambda_{l} t}\right|\left\|u_{0}\right\| .
$$

Thus it is sufficient to show that the modulus of the scalar quadrature error $\varepsilon(t ; \lambda)=$ $\left|Q(t ; \lambda)-e^{-\lambda t}\right|$ can be estimated as in the theorem (with $\left\|u_{0}\right\|=1$ ) for $\lambda \geq \lambda_{0}$. Recall that the trapezoidal rule has the error bound (1.11). Therefore, recalling that $\nu=1+\mathrm{i}$ and $\sigma(y)=2 \tau^{-1} \log (1 / y)$, we have

$$
\varepsilon(t ; \lambda) \leq \frac{e^{-\gamma t}}{8 N^{2}} \int_{0}^{1}\left|f^{\prime \prime}(y)\right| d y,
$$

where, from (1.9) and (1.6),

$$
f(y)=2(\pi \tau)^{-1} y^{-1+1 / \alpha} \operatorname{Im}\left(\nu e^{-\mathrm{i} \sigma(y) t} w(\gamma+\nu \sigma(y)), \quad \text { with } w(z)=(\lambda-z)^{-1} .\right.
$$

To bound the integral, we write, using $-\sigma(y) t=\log y / \alpha$,

$f(y)=2(\pi \tau)^{-1} \operatorname{Im}(\nu F(y)), \quad$ where $F(y)=y^{-1+\nu / \alpha} W(y), W(y)=w(\gamma+\nu \sigma(y))$,

and find that

$$
F^{\prime \prime}(y)=y^{-3+\nu / \alpha}\left(\left(-1+\frac{\nu}{\alpha}\right)\left(-2+\frac{\nu}{\alpha}\right) W(y)+2\left(-1+\frac{\nu}{\alpha}\right) y W^{\prime}(y)+y^{2} W^{\prime \prime}(y)\right) .
$$


Initially we assume $t>\tau$, corresponding to $\alpha<1 / 2$. In this case

$$
\left|F^{\prime \prime}(y)\right| \leq C y^{-3+1 / \alpha}\left(\alpha^{-2}|W(y)|+\alpha^{-1} y\left|W^{\prime}(y)\right|+y^{2}\left|W^{\prime \prime}(y)\right|\right) .
$$

Because $\sigma^{\prime}(y)=-2(\tau y)^{-1}$, we have

$$
\begin{aligned}
W^{\prime}(y) & =-2 \nu(\tau y)^{-1} w^{\prime}(\gamma+\nu \sigma(y)) \\
W^{\prime \prime}(y) & =4 \nu^{2}(\tau y)^{-2} w^{\prime \prime}(\gamma+\nu \sigma(y))+2 \nu \tau^{-1} y^{-2} w^{\prime}(\gamma+\nu \sigma(y)),
\end{aligned}
$$

so that, because $\left|w^{(j)}(\gamma+\nu \sigma)\right| \leq C(\sigma+1)^{-1-j}$ for $\sigma \geq 0, \lambda \geq \lambda_{0}>\gamma \geq 0, j=$ $0,1,2$,

$$
\begin{gathered}
|W(y)| \leq C(\sigma(y)+1)^{-1}, \quad y\left|W^{\prime}(y)\right| \leq C \tau^{-1}(\sigma(y)+1)^{-2} \\
y^{2}\left|W^{\prime \prime}(y)\right| \leq C\left(\tau^{-2}(\sigma(y)+1)^{-3}+\tau^{-1}(\sigma(y)+1)^{-2}\right) .
\end{gathered}
$$

Using also $\alpha t=\frac{1}{2} \tau$, it follows that

$$
\begin{aligned}
\left|f^{\prime \prime}(y)\right| \leq & C \tau^{-1}\left|F^{\prime \prime}(y)\right| \\
\leq & C y^{-3+1 / \alpha} \tau^{-1}\left(\alpha^{-2}(\sigma(y)+1)^{-1}\right. \\
& \left.\quad+\alpha^{-1} \tau^{-1}(\sigma(y)+1)^{-2}+\tau^{-2}(\sigma(y)+1)^{-3}\right) \\
& \leq C y^{-3+1 / \alpha} \tau^{-3}\left(t^{2}(\sigma(y)+1)^{-1}+(\sigma(y)+1)^{-2}\right),
\end{aligned}
$$

where in the last step we used the geometric-arithmetic mean inequality to bound the middle term in the large parentheses by the other two, and then bounded $(\sigma+1)^{-3}$ by $(\sigma+1)^{-2}$. Hence

$$
\int_{0}^{1}\left|f^{\prime \prime}(y)\right| d y \leq C \tau^{-3}\left(t^{2} J_{1}+J_{2}\right)
$$

where, since $y=e^{-\alpha \sigma t}$ and $\alpha t=\frac{1}{2} \tau$,

$$
J_{j}=\int_{0}^{1} y^{-3+1 / \alpha}(\sigma(y)+1)^{-j} d y=\frac{\tau}{2} \int_{0}^{\infty} e^{-(t-\tau) \sigma}(\sigma+1)^{-j} d \sigma, \quad j=1,2 .
$$

As in (2.4) we see that

$$
J_{1} \leq C \tau\left(\frac{1}{1+t-\tau}+\log _{+} \frac{1}{t-\tau}\right)
$$

and, by a simpler argument, $J_{2} \leq C \tau /(1+t-\tau)$, so that altogether

$$
\int_{0}^{1}\left|f^{\prime \prime}(y)\right| d y \leq C \tau^{-2}\left(\frac{1+t^{2}}{1+t-\tau}+t^{2} \log _{+} \frac{1}{t-\tau}\right)
$$

This completes the proof for $t>\tau$.

For $t \leq \tau$, i.e., for $\alpha \geq 1 / 2$, we see that the integral of $\left|f^{\prime \prime}(y)\right|$ fails to converge at 0 . For $1 / 2 \leq \alpha<1$ we see that $f(y)$ vanishes at $y=0$, as a result of which we can take the point of view that the trapezoidal rule for the integral of $f$ is used just on the interval $\left[y_{1}, 1\right]$, with an error of $\frac{1}{2} N^{-1}$ in the weight at $y_{1}=N^{-1}$, while on the interval $\left[0, y_{1}\right]$ the integral is approximated by zero. This leads to replacing the bound (1.11) by a new bound,

$$
\left|T_{N} f-I f\right| \leq \frac{1}{8 N^{2}} \int_{y_{1}}^{1}\left|f^{\prime \prime}(y)\right| d y+\frac{1}{2 N}\left|f\left(y_{1}\right)\right|+\int_{0}^{y_{1}}|f(y)| d y .
$$


The same bound can also be used for $\alpha \geq 1$ because we are ignoring the singularity at $y=0$ in the approximate integration in (1.9). This time we get, instead of (2.6),

$$
\left|F^{\prime \prime}(y)\right| \leq C y^{-3+1 / \alpha}\left(|W(y)|+y\left|W^{\prime}(y)\right|+y^{2}\left|W^{\prime \prime}(y)\right|\right),
$$

and hence the analogue of $(2.8)$ for the interval $\left(y_{1}, 1\right)$ is

$$
\int_{y_{1}}^{1}\left|f^{\prime \prime}(y)\right| d y \leq C \tau^{-3}\left(\tau^{2} J_{1 N}+J_{2 N}\right), \quad J_{j N}=\int_{y_{1}}^{1} y^{-3+1 / \alpha}(\sigma(y)+1)^{-j} d y,
$$

or, recalling that $\sigma_{1}=\sigma\left(y_{1}\right)=2 \tau^{-1} \log N$, and setting $\delta=\tau-t$,

$$
J_{j N}=\frac{\tau}{2} \int_{0}^{\sigma_{1}} \frac{e^{\delta \sigma}}{(\sigma+1)^{j}} d \sigma=\frac{\tau}{2} N^{2-2 t / \tau} \int_{0}^{\sigma_{1}} \frac{e^{-\delta\left(\sigma_{1}-\sigma\right)}}{(\sigma+1)^{j}} d \sigma,
$$

where we used $e^{\delta \sigma_{1}}=N^{2-2 t / \tau}$. For $j=2$ we have

$$
\int_{0}^{\sigma_{1}} \frac{e^{-\delta\left(\sigma_{1}-\sigma\right)}}{(\sigma+1)^{j}} d \sigma \leq \int_{0}^{\infty} \frac{1}{(\sigma+1)^{2}} d \sigma=1 .
$$

For $j=1$ and $t=\tau$,

$$
J_{1 N}=\frac{\tau}{2} \int_{0}^{\sigma_{1}} \frac{d \sigma}{\sigma+1}=\frac{\tau}{2} \log \left(\sigma_{1}+1\right) \leq C \tau\left(\log \log N+\log _{+} \frac{1}{\tau}\right) .
$$

To complete the proof for $t=\tau$, we note that since $w(\gamma+\nu \sigma)$ is bounded for $\sigma \geq 0$, we have $|f(y)| \leq C \tau^{-1} y$ and hence

$$
\frac{1}{2 N}\left|f\left(y_{1}\right)\right|+\int_{0}^{y_{1}}|f(y)| d y \leq C \frac{1}{N^{2}} \frac{1}{\tau} \leq C \frac{1}{N^{2}} \frac{1+\tau^{2}}{\tau^{2}} .
$$

For $j=1$ and $t<\tau$ we find first for $1 / \delta \geq \sigma_{1}$ that

$$
\int_{0}^{\sigma_{1}} \frac{e^{-\delta\left(\sigma_{1}-\sigma\right)}}{(\sigma+1)^{j}} d \sigma \leq \int_{0}^{1 / \delta} \frac{d \sigma}{\sigma+1}=\log \left(\delta^{-1}+1\right)
$$

for $1 / \delta<\sigma_{1}$ we have to add the term

$$
\int_{1 / \delta}^{\sigma_{1}} \frac{e^{-\delta\left(\sigma_{1}-\sigma\right)}}{\sigma+1} d \sigma \leq \delta \int_{1 / \delta}^{\sigma_{1}} e^{-\delta\left(\sigma_{1}-\sigma\right)} d \sigma \leq 1 .
$$

Thus

$$
\int_{y_{1}}^{1}\left|f^{\prime \prime}(y)\right| d y \leq C N^{2-2 t / \tau}\left(\frac{1}{\tau^{2}}+1+\log _{+} \frac{1}{\tau-t}\right) .
$$

Since $|f(y)| \leq C \tau^{-1} y^{-1+1 / \alpha}(\sigma(y)+1)^{-1}$ we also have

$$
\frac{1}{2 N}\left|f\left(y_{1}\right)\right|+\int_{0}^{y_{1}}|f(y)| d y \leq C N^{-2 t / \tau}\left(\frac{1}{\tau}+1+\log _{+} \frac{1}{t}\right),
$$

where for the second term we used

$$
\begin{aligned}
\frac{2}{\tau} \int_{0}^{y_{1}} \frac{y^{-1+1 / \alpha}}{\sigma(y)+1} d y & =\int_{\sigma_{1}}^{\infty} \frac{e^{-\sigma t}}{\sigma+1} d \sigma=e^{-\sigma_{1} t} \int_{0}^{\infty} \frac{e^{-x t}}{x+\sigma_{1}+1} d x \\
& \leq N^{-2 t / \tau} \int_{0}^{\infty} \frac{e^{-x t}}{x+1} d x \leq C N^{-2 t / \tau}\left(1+\log _{+} \frac{1}{t}\right)
\end{aligned}
$$

with the last step following from (2.4). Collecting the estimates, the desired result for $\alpha>1 / 2$, or $t<\tau$, follows. 
We remark that if instead of $U(t)$ we use the modified approximate solution $U_{\epsilon}(t)$ introduced after the proof of Proposition 2.1, then the error bound takes the simpler form

$$
\left\|U_{\epsilon}(t)-u(t)\right\| \leq C e^{-\gamma t}\left\|A^{\epsilon} u_{0}\right\| \begin{cases}\frac{1}{N^{2}} \frac{1+t^{2}}{\tau^{2}}, & t \geq \tau, \\ \frac{1}{N^{2 t / \tau}} \frac{1+\tau^{2}}{\tau^{2}}, & t \leq \tau .\end{cases}
$$

In fact, for $t \geq \tau$, we have instead of (2.7)

$$
\left|f^{\prime \prime}(y)\right| \leq C y^{-3+1 / \alpha} \tau^{-3}\left(t^{2}+1\right)(\sigma(y)+1)^{-1-\epsilon}
$$

and hence (cf. (2.8) and (2.9))

$$
\int_{0}^{1}\left|f^{\prime \prime}(y)\right| d y \leq C \tau^{-2}\left(t^{2}+1\right) \int_{0}^{\infty} e^{-\sigma(t-\tau)}(\sigma+1)^{-1-\epsilon} d \sigma \leq C_{\epsilon} \tau^{-2}\left(t^{2}+1\right) .
$$

The estimate for $t \leq \tau$ is derived similarly.

Let us now comment on our above choice of the slope 1 in $\Gamma=\Gamma_{\gamma}$. In fact, choosing instead $\Gamma_{\gamma, s}=\{z=\gamma+\sigma \pm i s \sigma ; 0 \leq \sigma<\infty\}$, it is easy to see that the bounds for $\left\|w^{(j)}(z)\right\|$ have to be multiplied by $(1+1 / s)^{j+1}$. On the other hand, from the proof of Theorem 2.2 we see that the factors $\nu=1+\mathrm{i}$ in front of $f(y)$ and in $W^{\prime}$ and $W^{\prime \prime}$ should now be replaced by $\nu_{s}=1+\mathrm{i} s$. The total change in the error bound in Theorem 2.2 would be a factor $(s+1 / s)^{3}$, and we therefore see that $s$ should be chosen neither too large nor too small. A natural choice is $s=1$.

We remark that the error estimate of Theorem 2.2 holds not only for the trapezoidal rule but also for any other composite rule on the uniform partition $y_{j}, j=$ $0, \ldots, N$, which is exact for linear functions. In particular, it holds for the Simpson rule, but with less than optimal accuracy.

Before turning to the full treatment of Simpson's rule, we pause to exhibit some numerical values of the quadrature errors $\varepsilon_{N}=\varepsilon_{N}(t ; \lambda)=\left|Q(t ; \lambda)-e^{-\lambda t}\right|$, which illustrate the behavior of our time discretization method based on the trapezoidal rule. Table 1 shows these errors $\varepsilon_{N}$ for $\tau=1, \lambda=1$, and $\gamma=0$, with $N=20,40,80$ and 160, and Table 2 the corresponding errors when $\tau=1, \lambda=1, \gamma=0.75$. Here $\rho_{N}=\log _{2}\left(\varepsilon_{N / 2} / \varepsilon_{N}\right)$ is the local convergence rate.

We observe that the predicted $O\left(N^{-2}\right)$ asymptotic convergence rate for $t>\tau=1$ is very clear in the tables. For values of $t$ smaller than $\tau$ the accuracy and the order of convergence deteriorate, broadly in line with the predictions of Theorem 2.2, but oscillations in the error prevent a clear determination of orders of convergence. We also note that the errors at $t \approx \tau$ are smaller for $\gamma=0$ than for $\gamma=0.75$, but that the situation is reversed for large values of $t$, which is consistent with the error bounds of Theorem 2.2.

In Table 3 we show the effect of varying $\tau$, by repeating the calculation now with $\tau=\frac{1}{2}, \gamma=0$. We see, on the one hand, that the $O\left(N^{-2}\right)$ convergence rate now persists, as it should, for $\tau>\frac{1}{2}$. On the other hand the absolute errors for $1 \leq \tau<2$ are larger than we observed in Table 1. Again this is broadly in line with the predictions of the theorem, given the appearance of the $\tau^{-2}$ terms in the error bounds. The results in these tables and this discussion motivate our suggestion that the results for a fixed value of $\tau$ be used only for a limited range of $t$ values, such as $\tau \leq t \leq 2 \tau$ for the case $\gamma=0$.

We now turn to the case when the time discretization is accomplished by Simpson's rule. We first remark that the stability result of Proposition 2.1 remains valid 
TABle 1. Time discretization errors for trapezoidal rule with $\tau=$ $1, \lambda=1, \gamma=0$.

\begin{tabular}{cccccccc}
\hline \hline$t$ & $\varepsilon_{20}$ & $\varepsilon_{40}$ & $\rho_{40}$ & $\varepsilon_{80}$ & $\rho_{80}$ & $\varepsilon_{160}$ & $\rho_{160}$ \\
\hline \hline 0.2 & $0.253 \mathrm{E}-01$ & $0.128 \mathrm{E}-01$ & 0.98 & $0.543 \mathrm{E}-02$ & 1.24 & $0.130 \mathrm{E}-02$ & 2.06 \\
0.4 & $0.197 \mathrm{E}-02$ & $0.173 \mathrm{E}-02$ & 0.19 & $0.950 \mathrm{E}-03$ & 0.86 & $0.371 \mathrm{E}-03$ & 1.36 \\
0.6 & $0.172 \mathrm{E}-03$ & $0.897 \mathrm{E}-04$ & 0.94 & $0.964 \mathrm{E}-04$ & -0.10 & $0.443 \mathrm{E}-04$ & 1.12 \\
0.8 & $0.365 \mathrm{E}-03$ & $0.993 \mathrm{E}-04$ & 1.88 & $0.154 \mathrm{E}-04$ & 2.69 & $0.552 \mathrm{E}-06$ & 4.80 \\
\hline 1.0 & $0.150 \mathrm{E}-03$ & $0.266 \mathrm{E}-04$ & 2.50 & $0.685 \mathrm{E}-05$ & 1.96 & $0.225 \mathrm{E}-05$ & 1.60 \\
1.2 & $0.151 \mathrm{E}-04$ & $0.611 \mathrm{E}-05$ & 1.30 & $0.195 \mathrm{E}-05$ & 1.64 & $0.422 \mathrm{E}-06$ & 2.21 \\
1.4 & $0.806 \mathrm{E}-04$ & $0.195 \mathrm{E}-04$ & 2.05 & $0.500 \mathrm{E}-05$ & 1.96 & $0.125 \mathrm{E}-05$ & 2.00 \\
1.6 & $0.185 \mathrm{E}-03$ & $0.465 \mathrm{E}-04$ & 1.99 & $0.116 \mathrm{E}-04$ & 2.00 & $0.290 \mathrm{E}-05$ & 2.00 \\
1.8 & $0.292 \mathrm{E}-03$ & $0.730 \mathrm{E}-04$ & 2.00 & $0.182 \mathrm{E}-04$ & 2.00 & $0.456 \mathrm{E}-05$ & 2.00 \\
2.0 & $0.398 \mathrm{E}-03$ & $0.995 \mathrm{E}-04$ & 2.00 & $0.249 \mathrm{E}-04$ & 2.00 & $0.622 \mathrm{E}-05$ & 2.00 \\
\hline 3.0 & $0.929 \mathrm{E}-03$ & $0.232 \mathrm{E}-03$ & 2.00 & $0.580 \mathrm{E}-04$ & 2.00 & $0.145 \mathrm{E}-04$ & 2.00 \\
4.0 & $0.146 \mathrm{E}-02$ & $0.365 \mathrm{E}-03$ & 2.00 & $0.912 \mathrm{E}-04$ & 2.00 & $0.228 \mathrm{E}-04$ & 2.00 \\
5.0 & $0.199 \mathrm{E}-02$ & $0.498 \mathrm{E}-03$ & 2.00 & $0.124 \mathrm{E}-03$ & 2.00 & $0.311 \mathrm{E}-04$ & 2.00 \\
6.0 & $0.253 \mathrm{E}-02$ & $0.630 \mathrm{E}-03$ & 2.00 & $0.158 \mathrm{E}-03$ & 2.00 & $0.394 \mathrm{E}-04$ & 2.00 \\
\hline \hline
\end{tabular}

TABLE 2. Time discretization errors for trapezoidal rule with $\tau=$ $1, \lambda=1, \gamma=0.75$.

\begin{tabular}{cccccccc}
\hline \hline$t$ & $\varepsilon_{20}$ & $\varepsilon_{40}$ & $\rho_{40}$ & $\varepsilon_{80}$ & $\rho_{80}$ & $\varepsilon_{160}$ & $\rho_{160}$ \\
\hline \hline 0.2 & $0.282 \mathrm{E}-01$ & $0.127 \mathrm{E}-01$ & 1.15 & $0.524 \mathrm{E}-02$ & 1.28 & $0.139 \mathrm{E}-02$ & 1.92 \\
0.4 & $0.438 \mathrm{E}-02$ & $0.219 \mathrm{E}-03$ & 4.32 & $0.327 \mathrm{E}-03$ & -0.58 & $0.185 \mathrm{E}-03$ & 0.82 \\
0.6 & $0.456 \mathrm{E}-02$ & $0.123 \mathrm{E}-02$ & 1.89 & $0.353 \mathrm{E}-03$ & 1.80 & $0.101 \mathrm{E}-03$ & 1.80 \\
0.8 & $0.404 \mathrm{E}-02$ & $0.102 \mathrm{E}-02$ & 1.98 & $0.252 \mathrm{E}-03$ & 2.03 & $0.611 \mathrm{E}-04$ & 2.04 \\
\hline 1.0 & $0.323 \mathrm{E}-02$ & $0.811 \mathrm{E}-03$ & 2.00 & $0.203 \mathrm{E}-03$ & 2.00 & $0.510 \mathrm{E}-04$ & 1.99 \\
1.2 & $0.260 \mathrm{E}-02$ & $0.657 \mathrm{E}-03$ & 1.98 & $0.165 \mathrm{E}-03$ & 2.00 & $0.411 \mathrm{E}-04$ & 2.00 \\
1.4 & $0.209 \mathrm{E}-02$ & $0.529 \mathrm{E}-03$ & 1.99 & $0.132 \mathrm{E}-03$ & 2.00 & $0.331 \mathrm{E}-04$ & 2.00 \\
1.6 & $0.168 \mathrm{E}-02$ & $0.423 \mathrm{E}-03$ & 1.99 & $0.106 \mathrm{E}-03$ & 2.00 & $0.265 \mathrm{E}-04$ & 2.00 \\
1.8 & $0.133 \mathrm{E}-02$ & $0.337 \mathrm{E}-03$ & 1.99 & $0.842 \mathrm{E}-04$ & 2.00 & $0.211 \mathrm{E}-04$ & 2.00 \\
2.0 & $0.105 \mathrm{E}-02$ & $0.266 \mathrm{E}-03$ & 1.99 & $0.666 \mathrm{E}-04$ & 2.00 & $0.166 \mathrm{E}-04$ & 2.00 \\
\hline 3.0 & $0.275 \mathrm{E}-03$ & $0.698 \mathrm{E}-04$ & 1.98 & $0.175 \mathrm{E}-04$ & 2.00 & $0.437 \mathrm{E}-05$ & 2.00 \\
4.0 & $0.246 \mathrm{E}-04$ & $0.655 \mathrm{E}-05$ & 1.91 & $0.165 \mathrm{E}-05$ & 1.99 & $0.412 \mathrm{E}-06$ & 2.00 \\
5.0 & $0.382 \mathrm{E}-04$ & $0.938 \mathrm{E}-05$ & 2.02 & $0.234 \mathrm{E}-05$ & 2.00 & $0.585 \mathrm{E}-06$ & 2.00 \\
6.0 & $0.416 \mathrm{E}-04$ & $0.103 \mathrm{E}-04$ & 2.01 & $0.258 \mathrm{E}-05$ & 2.00 & $0.645 \mathrm{E}-06$ & 2.00 \\
\hline \hline
\end{tabular}

in this situation, since the points $\sigma_{j}$ are still defined by (1.12) and the weights $\omega_{j}(t)$ are now bounded by $\frac{4}{3}$ times those in (1.13). For the error we now have the following estimate.

Theorem 2.3. Let $\tau>0$, and assume that the eigenvalues of $A$ are bounded below by $\lambda_{0}$, and that $0 \leq \gamma<\lambda_{0}$. Assume that the quadrature approximation $U(t)$ is obtained by applying Simpson's rule (1.14) (with the $y_{0}$ term omitted) to the integral (1.5), where $g(t ; \sigma)$ is defined by (1.6) and $\alpha$ is chosen so that $\alpha t=\tau / 4$. Then there 
TABLE 3. Time discretization errors for trapezoidal rule with $\tau=$ $1 / 2, \lambda=1, \gamma=0$.

\begin{tabular}{cccccccc}
\hline \hline$t$ & $\varepsilon_{20}$ & $\varepsilon_{40}$ & $\rho_{40}$ & $\varepsilon_{80}$ & $\rho_{80}$ & $\varepsilon_{160}$ & $\rho_{160}$ \\
\hline \hline 0.2 & $0.301 \mathrm{E}-03$ & $0.129 \mathrm{E}-02$ & -2.09 & $0.838 \mathrm{E}-03$ & 0.62 & $0.347 \mathrm{E}-03$ & 1.27 \\
0.4 & $0.166 \mathrm{E}-02$ & $0.424 \mathrm{E}-03$ & 1.97 & $0.969 \mathrm{E}-04$ & 2.13 & $0.210 \mathrm{E}-04$ & 2.21 \\
\hline 0.6 & $0.110 \mathrm{E}-02$ & $0.278 \mathrm{E}-03$ & 1.99 & $0.699 \mathrm{E}-04$ & 1.99 & $0.174 \mathrm{E}-04$ & 2.01 \\
0.8 & $0.689 \mathrm{E}-03$ & $0.172 \mathrm{E}-03$ & 2.00 & $0.431 \mathrm{E}-04$ & 2.00 & $0.108 \mathrm{E}-04$ & 2.00 \\
1.0 & $0.263 \mathrm{E}-03$ & $0.662 \mathrm{E}-04$ & 1.99 & $0.166 \mathrm{E}-04$ & 2.00 & $0.414 \mathrm{E}-05$ & 2.00 \\
1.2 & $0.161 \mathrm{E}-03$ & $0.399 \mathrm{E}-04$ & 2.01 & $0.996 \mathrm{E}-05$ & 2.00 & $0.249 \mathrm{E}-05$ & 2.00 \\
1.4 & $0.585 \mathrm{E}-03$ & $0.146 \mathrm{E}-03$ & 2.00 & $0.365 \mathrm{E}-04$ & 2.00 & $0.912 \mathrm{E}-05$ & 2.00 \\
1.6 & $0.101 \mathrm{E}-02$ & $0.252 \mathrm{E}-03$ & 2.00 & $0.630 \mathrm{E}-04$ & 2.00 & $0.158 \mathrm{E}-04$ & 2.00 \\
1.8 & $0.143 \mathrm{E}-02$ & $0.358 \mathrm{E}-03$ & 2.00 & $0.895 \mathrm{E}-04$ & 2.00 & $0.224 \mathrm{E}-04$ & 2.00 \\
2.0 & $0.186 \mathrm{E}-02$ & $0.464 \mathrm{E}-03$ & 2.00 & $0.116 \mathrm{E}-03$ & 2.00 & $0.290 \mathrm{E}-04$ & 2.00 \\
\hline \hline
\end{tabular}

exists $C=C\left(\lambda_{0}-\gamma\right)>0$, such that

$$
\|U(t)-u(t)\| \leq C\left\|u_{0}\right\| e^{-\gamma t} \begin{cases}\frac{1}{N^{4}}\left(\frac{1+t^{4}}{\tau^{4}(1+t-\tau)}+\frac{t^{4}}{\tau^{4}} \log _{+} \frac{1}{t-\tau}\right), & t>\tau, \\ \frac{1}{N^{4}}\left(\log \log N+\frac{1}{\tau^{4}}+\log _{+} \frac{1}{\tau}\right), \quad N \geq 3, & t=\tau, \\ \frac{1}{N^{4 t / \tau}}\left(\frac{1+\tau^{4}}{\tau^{4}}+\log _{+} \frac{1}{\tau-t}+\log _{+} \frac{1}{t}\right), & 0<t<\tau .\end{cases}
$$

Proof. With $f(u)$ and $F(u)$ as earlier, we have this time

$$
\varepsilon(t ; \lambda) \leq \frac{C e^{-\gamma t}}{N^{4}} \int_{0}^{1}\left|f^{(i v)}(y)\right| d y .
$$

For $t>\tau$, or $\alpha<1 / 4,(2.6)$ is replaced by

$$
\left|F^{(i v)}(y)\right| \leq C y^{-5+1 / \alpha} \sum_{j=0}^{4} \alpha^{j-4} y^{j}\left|W^{(j)}(y)\right| .
$$

Here

$$
y^{j}\left|W^{(j)}(y)\right| \leq C \sum_{l \leq j} \tau^{-l}\left|w^{(l)}(\gamma+\nu \sigma(y))\right| \leq C \sum_{l \leq j} \tau^{-l}(\sigma(y)+1)^{-l-1}, \quad \text { for } j \leq 4,
$$

so that

$$
\left|F^{(i v)}(y)\right| \leq C y^{-5+1 / \alpha} \tau^{-4}\left(t^{4}(\sigma(y)+1)^{-1}+(\sigma(y)+1)^{-2}\right) .
$$

We conclude that, with

$$
J_{j}=\int_{0}^{1} y^{-5+1 / \alpha}(\sigma(y)+1)^{-j} d y, \quad j=1,2,
$$

we have

$$
\int_{0}^{1}\left|f^{(i v)}(y)\right| d y \leq C \tau^{-5}\left(t^{4} J_{1}+J_{2}\right),
$$

and the argument is completed as before. 
TABle 4. Time discretization errors for Simpson's rule with $\tau=$ $1, \lambda=1, \gamma=0$.

\begin{tabular}{cccccccc}
\hline \hline$t$ & $\varepsilon_{20}$ & $\varepsilon_{40}$ & $\rho_{40}$ & $\varepsilon_{80}$ & $\rho_{80}$ & $\varepsilon_{160}$ & $\rho_{160}$ \\
\hline \hline 0.2 & $0.278 \mathrm{E}-02$ & $0.161 \mathrm{E}-02$ & 0.78 & $0.688 \mathrm{E}-03$ & 1.23 & $0.183 \mathrm{E}-03$ & 1.91 \\
0.4 & $0.233 \mathrm{E}-03$ & $0.131 \mathrm{E}-04$ & 4.15 & $0.120 \mathrm{E}-04$ & 0.13 & $0.436 \mathrm{E}-05$ & 1.45 \\
0.6 & $0.983 \mathrm{E}-05$ & $0.334 \mathrm{E}-05$ & 1.56 & $0.619 \mathrm{E}-06$ & 2.43 & $0.822 \mathrm{E}-07$ & 2.91 \\
0.8 & $0.330 \mathrm{E}-04$ & $0.390 \mathrm{E}-07$ & 9.73 & $0.481 \mathrm{E}-07$ & -0.30 & $0.566 \mathrm{E}-08$ & 3.09 \\
\hline 1.0 & $0.222 \mathrm{E}-04$ & $0.469 \mathrm{E}-06$ & 5.56 & $0.329 \mathrm{E}-07$ & 3.83 & $0.226 \mathrm{E}-08$ & 3.87 \\
1.2 & $0.208 \mathrm{E}-04$ & $0.389 \mathrm{E}-06$ & 5.74 & $0.341 \mathrm{E}-07$ & 3.51 & $0.204 \mathrm{E}-08$ & 4.06 \\
1.4 & $0.184 \mathrm{E}-04$ & $0.438 \mathrm{E}-06$ & 5.40 & $0.344 \mathrm{E}-07$ & 3.67 & $0.211 \mathrm{E}-08$ & 4.03 \\
1.6 & $0.176 \mathrm{E}-04$ & $0.511 \mathrm{E}-06$ & 5.11 & $0.379 \mathrm{E}-07$ & 3.76 & $0.233 \mathrm{E}-08$ & 4.02 \\
1.8 & $0.177 \mathrm{E}-04$ & $0.626 \mathrm{E}-06$ & 4.83 & $0.440 \mathrm{E}-07$ & 3.83 & $0.271 \mathrm{E}-08$ & 4.02 \\
2.0 & $0.187 \mathrm{E}-04$ & $0.779 \mathrm{E}-06$ & 4.59 & $0.527 \mathrm{E}-07$ & 3.89 & $0.327 \mathrm{E}-08$ & 4.01 \\
\hline \hline
\end{tabular}

For $t \leq \tau$, corresponding to $\alpha \geq \frac{1}{4}$, we use instead of (1.15) the estimate

$$
\left|S_{N} f-I f\right| \leq \frac{C}{N^{4}} \int_{y_{2}}^{1}\left|f^{(i v)}(y)\right| d y+\frac{4}{3 N}\left|f\left(y_{1}\right)\right|+\frac{1}{3 N}\left|f\left(y_{2}\right)\right|+\int_{0}^{\frac{2}{N}}|f(y)| d y,
$$

which is the appropriate bound for Simpson's rule for the interval $\left[y_{2}, 1\right]$, together with estimation by zero in the interval $\left[0, y_{2}\right]$. The remainder of the proof follows in the same manner as in Theorem 2.2.

We complete this section by presenting in Table 4 the analogue for Simpson's rule of Table 1. Thus Table 4 shows the quadrature error $\varepsilon_{N}=\left|Q_{\tau}(t ; \lambda)-e^{-\lambda t}\right|$ for $\tau=1, \lambda=1$, and $\gamma=0$, with $N=20,40,80$, and 160, for Simpson's rule. For $t>\tau$ the predicted $O\left(N^{-4}\right)$ accuracy is clearly seen, and the errors are correspondingly small, while for $t<\tau$ the reduced rate of convergence is particularly clear.

It should be emphasized that in the present method Simpson's rule with a given value of $N$ requires exactly the same computational effort as the trapezoidal rule. A comparison between Tables 1 and 4 will convince the reader that Simpson's rule is superior. The numerical results in Table 4 also suggest that in practical calculations, such as the finite-element calculations of the next section, a value of, say, $N=40$ in Simpson's rule should be more than adequate in most cases.

\section{Applichtion to the finite element method}

We now consider the application of our quadrature based methods to the "parabolic" equation in the piecewise linear space $V_{h}$ which has been obtained by discretization in the space of the initial-boundary value problem (1.16), i.e., the semidiscrete problem (1.18). With our earlier definitions, this may be written

$$
u_{h, t}+A_{h} u_{h}=0, \quad \text { for } t>0, \quad \text { with } u_{h}(0)=P_{h} u_{0} .
$$

As explained in the introduction, our fully discrete approximation $U_{h}(t) \in V_{h}$ is then obtained by the application of one of our quadrature methods to the integral (1.5), where now $g(t ; \sigma)$ in (1.6) is replaced by $g_{h}(t ; \sigma)$, defined in terms of the finite element approximation $w_{h}(z)$ from $(1.17)$ of the solution $w(z)$ of the elliptic 
equation in (1.4), so that

$$
U_{h}(t)=Q\left(t ; A_{h}\right) P_{h} u_{0}=\sum_{j=1}^{N} \omega_{j}(t) g_{h}\left(t ; \sigma_{j}\right), \quad g_{h}(t ; \sigma)=\frac{1}{\pi} \operatorname{Im}\left(\nu e^{-\mathrm{i} \sigma t} w_{h}(\gamma+\nu \sigma)\right) .
$$

The error $U_{h}-u$ may be handled by splitting it as

$$
U_{h}(t)-u(t)=\left(U_{h}(t)-u_{h}(t)\right)+\left(u_{h}(t)-u(t)\right) .
$$

If the trapezoidal rule is used for the time discretization, then we may apply Theorem 2.2 to the first part of the error, because the smallest eigenvalue $\lambda_{h, 1}$ of $A_{h}$ is bounded below by the smallest eigenvalue $\lambda_{1}$ of $A$. Hence, since $\left\|P_{h} u_{0}\right\| \leq\left\|u_{0}\right\|$, the first part of the error in (3.1) is bounded in norm by the error bound in Theorem 2.2. The second part of the error in (3.1) is bounded by (cf. [10])

$$
\left\|u_{h}(t)-u(t)\right\| \leq C h^{2} t^{-1} e^{-\gamma t}\left\|u_{0}\right\|, \quad \text { for } t>0,
$$

or, more generally, also for smoother initial data, by

$$
\left\|u_{h}(t)-u(t)\right\| \leq C h^{2} t^{-1+\epsilon} e^{-\gamma t}\left\|A^{\epsilon} u_{0}\right\|, \quad \text { for } t>0, \quad 0 \leq \epsilon \leq 1 ;
$$

together these estimates yield complete error estimates for our method. We remark that for smooth initial data the error in the spatially semidiscrete equation is thus $O\left(h^{2}\right)$, uniformly down to $t=0$, but that the $O\left(N^{-2}\right)$ error bound in the quadrature method based on the trapezoidal rule holds only for $t>\tau$, even when initial data are smooth.

Similarly, for the case of Simpson's rule the error bound for $\left\|U_{h}(t)-u_{h}(t)\right\|$ is exactly as in Theorem 2.3, and the total error for $t>\tau$ is $O\left(N^{-4}+h^{2}\right)$.

We now give some illustrations, beginning with the spatially one-dimensional problem

$$
\begin{gathered}
u_{t}=u_{x x}, \quad \text { in }[0, \pi], \quad \text { with } u(0, t)=u(\pi, t)=0, \quad \text { for } t>0, \\
u(x, 0)=u_{0}(x), \quad \text { in }[0, \pi] .
\end{gathered}
$$

In Tables 5, 6, and 7 we exhibit errors $\varepsilon_{N, M}$ in the numerical results for the initial function $u_{0}(x)=(5 \pi / 2)^{-1 / 2}(\sin x+2 \sin 2 x)$ (with $\left\|u_{0}\right\|=1$ ) for, respectively, the trapezoidal rule, Simpson's rule, and, for comparison, the Crank-Nicolson method. In all cases the spatial discretization uses piecewise linear approximations on regular meshes, with $h=\pi / M$, and $N$ is chosen as 20,40,80, and 160 for both the trapezoidal rule and the Crank-Nicolson method (with time step $1 / N$ ), and as 10, 20, and 40 for Simpson's rule. In Tables 5 and 6 we choose $\tau=1$ and $\gamma=0$, and show results only for $\tau \leq t \leq 2 \tau$, which is the recommended way of using the method, and for the Crank-Nicolson method we restrict ourselves to $t=1$ and $t=2$. In Tables 5 and 7 , $\rho_{N, M}=\log _{2}\left(\varepsilon_{N / 2, M / 2} / \varepsilon_{N, M}\right)$, and in Table $6, \rho_{N, M}=\log _{2}\left(\varepsilon_{N / 2, M / 4} / \varepsilon_{N, M}\right)$. The results again show the expected $O\left(N^{-2}+h^{2}\right)=O\left(N^{-2}+M^{-2}\right)$ order of convergence for the trapezoidal and Crank-Nicolson cases and $\left.O\left(N^{-4}+h^{2}\right)=O\left(N^{-4}+M^{-2}\right)\right)$ for Simpson's rule. Note that the higher order of the discretization in Simpson's rule compared with the Crank-Nicolson method allows us to obtain accuracy of order $10^{-6}$ in the interval $[1,2]$ with a much coarser time discretization.

As our next illustration, we consider again the spatially one-dimensional problem (3.2), now with the nonsmooth initial function $u_{0}(x)=(\pi / 2)^{-1 / 2}$ for $\frac{\pi}{4} \leq x \leq \frac{3 \pi}{4}$, and $u_{0}(x)=0$ for other $x$ in $[0, \pi]$ (again with $\left\|u_{0}\right\|=1$ ). The analogues of Tables 5, 6, and 7 above are exhibited in Tables 8, 9, and 10. Since the Crank-Nicolson method is known not to deal well with nonsmooth data, we exhibit also the result 
TABle 5. Trapezoidal rule errors for $1 D$ heat equation with $\tau=$ $1, \gamma=0$.

\begin{tabular}{cccccccc}
\hline \hline$t$ & $\varepsilon_{20,50}$ & $\varepsilon_{40,100}$ & $\rho_{40,100}$ & $\varepsilon_{80,200}$ & $\rho_{80,200}$ & $\varepsilon_{160,400}$ & $\rho_{160,400}$ \\
\hline \hline 1.0 & $0.129 \mathrm{E}-03$ & $0.262 \mathrm{E}-04$ & 2.29 & $0.667 \mathrm{E}-05$ & 1.98 & $0.201 \mathrm{E}-05$ & 1.73 \\
1.2 & $0.796 \mathrm{E}-04$ & $0.192 \mathrm{E}-04$ & 2.05 & $0.481 \mathrm{E}-05$ & 1.99 & $0.121 \mathrm{E}-05$ & 1.99 \\
1.4 & $0.849 \mathrm{E}-04$ & $0.210 \mathrm{E}-04$ & 2.02 & $0.537 \mathrm{E}-05$ & 1.97 & $0.134 \mathrm{E}-05$ & 2.00 \\
1.6 & $0.123 \mathrm{E}-03$ & $0.311 \mathrm{E}-04$ & 1.98 & $0.775 \mathrm{E}-05$ & 2.00 & $0.194 \mathrm{E}-05$ & 2.00 \\
1.8 & $0.172 \mathrm{E}-03$ & $0.430 \mathrm{E}-04$ & 2.00 & $0.107 \mathrm{E}-04$ & 2.00 & $0.268 \mathrm{E}-05$ & 2.00 \\
2.0 & $0.223 \mathrm{E}-03$ & $0.557 \mathrm{E}-04$ & 2.00 & $0.139 \mathrm{E}-04$ & 2.00 & $0.348 \mathrm{E}-05$ & 2.00 \\
\hline \hline
\end{tabular}

TABLE 6. Simpson's rule errors for $1 D$ heat equation with $\tau=$ $1, \gamma=0$.

\begin{tabular}{cccccc}
\hline \hline$t$ & $\varepsilon_{10,25}$ & $\varepsilon_{20,100}$ & $\rho_{20,100}$ & $\varepsilon_{40,400}$ & $\rho_{40,400}$ \\
\hline \hline 1.0 & $0.216 \mathrm{E}-02$ & $0.320 \mathrm{E}-04$ & 6.08 & $0.184 \mathrm{E}-05$ & 4.12 \\
1.2 & $0.173 \mathrm{E}-02$ & $0.265 \mathrm{E}-04$ & 6.02 & $0.131 \mathrm{E}-05$ & 4.34 \\
1.4 & $0.136 \mathrm{E}-02$ & $0.226 \mathrm{E}-04$ & 5.91 & $0.113 \mathrm{E}-05$ & 4.32 \\
1.6 & $0.107 \mathrm{E}-02$ & $0.208 \mathrm{E}-04$ & 5.69 & $0.105 \mathrm{E}-05$ & 4.30 \\
1.8 & $0.836 \mathrm{E}-03$ & $0.197 \mathrm{E}-04$ & 5.41 & $0.103 \mathrm{E}-05$ & 4.26 \\
2.0 & $0.643 \mathrm{E}-03$ & $0.192 \mathrm{E}-04$ & 5.07 & $0.103 \mathrm{E}-05$ & 4.21 \\
\hline \hline
\end{tabular}

TABLE 7. Crank-Nicolson errors for $1 D$ heat equation.

\begin{tabular}{cccccccc}
\hline \hline$t$ & $\varepsilon_{20,50}$ & $\varepsilon_{40,100}$ & $\rho_{40,100}$ & $\varepsilon_{80,200}$ & $\rho_{80,200}$ & $\varepsilon_{160,400}$ & $\rho_{160,400}$ \\
\hline \hline 1.0 & $0.975 \mathrm{E}-03$ & $0.245 \mathrm{E}-03$ & 2.00 & $0.612 \mathrm{E}-04$ & 2.00 & $0.153 \mathrm{E}-04$ & 2.00 \\
2.0 & $0.145 \mathrm{E}-03$ & $0.363 \mathrm{E}-04$ & 2.00 & $0.908 \mathrm{E}-05$ & 2.00 & $0.227 \mathrm{E}-05$ & 2.00 \\
\hline \hline
\end{tabular}

TABLE 8. As in Table 5 with nonsmooth initial data.

\begin{tabular}{cccccccc}
\hline \hline$t$ & $\varepsilon_{20,50}$ & $\varepsilon_{40,100}$ & $\rho_{40,100}$ & $\varepsilon_{80,200}$ & $\rho_{80,200}$ & $\varepsilon_{160,400}$ & $\rho_{160,400}$ \\
\hline \hline 1.0 & $0.139 \mathrm{E}-03$ & $0.298 \mathrm{E}-04$ & 2.22 & $0.754 \mathrm{E}-05$ & 1.99 & $0.215 \mathrm{E}-05$ & 1.81 \\
1.2 & $0.709 \mathrm{E}-04$ & $0.191 \mathrm{E}-04$ & 1.89 & $0.499 \mathrm{E}-05$ & 1.94 & $0.122 \mathrm{E}-05$ & 2.04 \\
1.4 & $0.261 \mathrm{E}-04$ & $0.697 \mathrm{E}-05$ & 1.90 & $0.171 \mathrm{E}-05$ & 2.03 & $0.427 \mathrm{E}-06$ & 2.00 \\
1.6 & $0.454 \mathrm{E}-04$ & $0.113 \mathrm{E}-04$ & 2.01 & $0.281 \mathrm{E}-05$ & 2.00 & $0.701 \mathrm{E}-06$ & 2.00 \\
1.8 & $0.101 \mathrm{E}-03$ & $0.251 \mathrm{E}-04$ & 2.01 & $0.627 \mathrm{E}-05$ & 2.00 & $0.157 \mathrm{E}-05$ & 2.00 \\
2.0 & $0.159 \mathrm{E}-03$ & $0.395 \mathrm{E}-04$ & 2.01 & $0.988 \mathrm{E}-05$ & 2.00 & $0.247 \mathrm{E}-05$ & 2.00 \\
\hline \hline
\end{tabular}

obtained by the modification of using the backward Euler method for the first two time steps, which smooths the initial data (cf. [10]). As expected, the results in Tables 8 and 9 show the same behavior for nonsmooth as for smooth initial data. For the nonsmooth initial data the Simpson's rule results in Table 9 with $N=40$ compare very favorably with even the modified Crank-Nicolson results with 160 time steps. 
TABLE 9. As in Table 6 with nonsmooth initial data.

\begin{tabular}{cccccc}
\hline \hline$t$ & $\varepsilon_{10,25}$ & $\varepsilon_{20,100}$ & $\rho_{20,100}$ & $\varepsilon_{40,400}$ & $\rho_{40,400}$ \\
\hline \hline 1.0 & $0.204 \mathrm{E}-02$ & $0.274 \mathrm{E}-04$ & 6.22 & $0.127 \mathrm{E}-05$ & 4.43 \\
1.2 & $0.164 \mathrm{E}-02$ & $0.262 \mathrm{E}-04$ & 5.97 & $0.119 \mathrm{E}-05$ & 4.46 \\
1.4 & $0.129 \mathrm{E}-02$ & $0.242 \mathrm{E}-04$ & 5.74 & $0.116 \mathrm{E}-05$ & 4.38 \\
1.6 & $0.100 \mathrm{E}-02$ & $0.227 \mathrm{E}-04$ & 5.47 & $0.113 \mathrm{E}-05$ & 4.33 \\
1.8 & $0.776 \mathrm{E}-03$ & $0.216 \mathrm{E}-04$ & 5.16 & $0.111 \mathrm{E}-05$ & 4.28 \\
2.0 & $0.586 \mathrm{E}-03$ & $0.209 \mathrm{E}-04$ & 4.81 & $0.111 \mathrm{E}-05$ & 4.23 \\
\hline \hline
\end{tabular}

TABLE 10. Crank-Nicolson $1 D$ errors, without and with backward Euler smoothing.

\begin{tabular}{cccccccc}
\hline \hline$t$ & $\varepsilon_{20,50}$ & $\varepsilon_{40,100}$ & $\rho_{40,100}$ & $\varepsilon_{80,200}$ & $\rho_{80,200}$ & $\varepsilon_{160,400}$ & $\rho_{160,400}$ \\
\hline \hline 1.0 & $0.504 \mathrm{E}-01$ & $0.263 \mathrm{E}-01$ & 0.94 & $0.186 \mathrm{E}-01$ & 0.50 & $0.132 \mathrm{E}-01$ & 0.50 \\
2.0 & $0.367 \mathrm{E}-01$ & $0.168 \mathrm{E}-01$ & 1.12 & $0.119 \mathrm{E}-01$ & 0.50 & $0.843 \mathrm{E}-02$ & 0.50 \\
\hline 1.0 & $0.158 \mathrm{E}-02$ & $0.402 \mathrm{E}-03$ & 1.97 & $0.102 \mathrm{E}-03$ & 1.98 & $0.256 \mathrm{E}-04$ & 1.99 \\
2.0 & $0.499 \mathrm{E}-03$ & $0.128 \mathrm{E}-03$ & 1.96 & $0.325 \mathrm{E}-04$ & 1.98 & $0.818 \mathrm{E}-05$ & 1.99 \\
\hline \hline
\end{tabular}

TABle 11. Simpson's rule errors for $2 D$ heat equation, with $\tau=$ $1, \gamma=0$.

\begin{tabular}{cccc}
\hline \hline$t$ & $\varepsilon_{16,25}$ & $\varepsilon_{32,100}$ & $\rho_{32,100}$ \\
\hline \hline 1.0 & $0.335 \mathrm{E}-03$ & $0.209 \mathrm{E}-04$ & 4.00 \\
1.2 & $0.269 \mathrm{E}-03$ & $0.166 \mathrm{E}-04$ & 4.02 \\
1.4 & $0.212 \mathrm{E}-03$ & $0.131 \mathrm{E}-04$ & 4.02 \\
1.6 & $0.165 \mathrm{E}-03$ & $0.102 \mathrm{E}-04$ & 4.02 \\
1.8 & $0.129 \mathrm{E}-03$ & $0.804 \mathrm{E}-05$ & 4.00 \\
2.0 & $0.103 \mathrm{E}-03$ & $0.644 \mathrm{E}-05$ & 4.00 \\
\hline \hline
\end{tabular}

Finally, we consider the spatially two-dimensional problem version of the boundary value problem for the heat equation, with $\Omega=[0, \pi] \times[0, \pi]$,

$$
\begin{gathered}
u_{t}=\Delta u, \quad \text { in } \Omega, \quad u(x, y, t)=0, \quad \text { for }(x, y) \in \partial \Omega, \quad t>0, \\
u(x, y, 0)=u_{0}(x, y), \quad \text { in } \Omega,
\end{gathered}
$$

with (non-smooth) initial data $u_{0}(x, y)=\chi_{\left[\frac{\pi}{5}, \frac{4 \pi}{5}\right] \times\left[\frac{\pi}{5}, \frac{4 \pi}{5}\right]}(x, y)$. The finite element space for the spatial approximation is obtained by dividing $\Omega$ into $M \times M$ identical rectangles $(h=\pi / M)$, and using piecewise bilinear elements on this mesh. For the time discretization we adopt Simpson's rule and choose $\tau$ and $\gamma$ to have the values 1 and 0 , respectively.

In Table 11 we present the resulting $L_{2}(\Omega)$ errors for $N=32$ and $M=100$, and also (to allow us to check the predicted $O\left(h^{2}+N^{-4}\right)$ rate of convergence) $N=16$ and $M=25$. Evidently the rate of convergence and the absolute errors in the time interval $[\tau, 2 \tau]$ are both highly acceptable.

To give substance to the claim that this is a parallel method, the results in Table 11 were obtained (using the Yale Sparse Matrix Package [4], an efficient version of Gaussian elimination, with double complex arithmetic) on a 16-processor IBM SP2, 
TABLE 12. Computing time and speedup ratio for $2 D$ heat equation.

\begin{tabular}{ccccccc}
\hline \hline Problem Size & $P$ & 1 & 2 & 4 & 8 & 16 \\
\hline \hline$N=32$ & $T$ & 81.3 & 40.5 & 20.7 & 10.6 & 5.6 \\
$M=50$ & $\rho$ & & 2.00 & 1.95 & 1.95 & 1.89 \\
\hline$N=32$ & $T$ & 942 & 471 & 236 & 119 & 61 \\
$M=100$ & $\rho$ & & 2.00 & 2.00 & 1.98 & 1.94 \\
\hline \hline
\end{tabular}

whose main architectural feature is that it has 16 essentially identical processors, with no shared memory. The main part of the computation is of course the solution of the elliptic finite-element problem (1.17), for $z=z_{j}=\gamma+\nu \sigma_{j}, j=1, \ldots, N$. The $N$ such problems were first solved one after another on a single processor; then for comparison distributed between 2 processors, so that each processor had to solve only half the number of elliptic problems; then distributed over 4 processors, and so on. The total computer clock times (including overheads) required with 1, 2, 4, 8, and 16 processors are reported in Table 12 for $N=32$ and $M=100$, as well as $M=50$ for comparison. In Table 12 the labels $P, T$, and $\rho$ denote the number of processors, the total computer clock time in seconds, and the speedup ratio $T(P / 2) / T(P)$, respectively. When the size of the finite element problem changes from $M=50$ to 100 the speedup ratios become closer to two, reflecting the truism that the bigger the size of the distributed problems, the more effective the parallelization. Since the total time for the largest problem continues to decrease roughly in proportion to the number of processors for $P$ up to 16 , the parallelization has been successful, and indeed could profitably employ many more processors than the 16 currently available to us. A parallel implementation with more than 16 processors would be expected to be even more beneficial for parabolic problems in three space dimensions.

\section{REFERENCES}

1. P. J. Davis and P. Rabinowitz, Methods of Numerical Integration, Academic Press, New York, 1975. MR 56:7119

2. J. Douglas, Jr., J. E. Santos, D. Sheen, and L. S. Bennethum, Frequency domain treatment of one-dimensional scalar waves, Math. Mod. Meth. Appl. Sci. 3 (1993), 171-194. MR 94g:65111

3. J. Douglas, Jr., J. E. Santos, and D. Sheen, Approximation of scalar waves in the spacefrequency domain, Math. Mod. Meth. Appl. Sci 4 (1994), 509-531. MR 95e:65089

4. S. C. Eisenstat, Jr., H. E. Elman, N. H. Schultz, and A. H. Sherman, The (new) Yale Sparse Matrix Package, Elliptic Problem Solvers II (A. L. Schoenstadt and G. Birkhoff, eds.), Academic Press, New York, 1983, pp. 45-52. MR 85g:65007

5. E. Gallopoulos and Y. Saad, Efficient solution of parabolic equations by Krylov approximation methods, SIAM J. Sci. Statist. Comput. 13 (1992), 1236-1264. MR 93d:65085

6. M. Hochbruck and C. Lubich, On Krylov Subspace Approximations to the Matrix Exponential Operator, SIAM J. Numer. Anal. 34 (1997), 1911-1925. MR 98h:65018

7. C.-O. Lee, J. Lee, and D. Sheen, A frequency-domain method for finite element solutions of parabolic problems, RIM-GARC Preprint 97-41, Department of Mathematics, Seoul National University, 1997.

8. A. Pazy, Semigroups of Linear Operators and Applications to Partial Differential Equations, Springer-Verlag, New York, 1983. MR 85g:47061

9. D. Sheen and Y. Yeom, A frequency-domain parallel method for the numerical approximation of parabolic problems, RIM-GARC Preprint 96-38, Department of Mathematics, Seoul National University, 1996. 
10. V. Thomée, Galerkin Finite Element Methods for Parabolic Problems, Springer Series in Computational Mathematics, Vol. 25, Springer-Verlag, Berlin Heidelberg New York, 1997. MR 98m:65007

11. R. S. Varga, Functional Analysis and Approximation Theory in Numerical Analysis, Conference Board of the Mathematical Sciences Regional Conferences Series in Applied Mathematics, No. 3, SIAM, Philadelphia, PA, 1971. MR 46:9602

Department of Mathematics, Seoul National University, Seoul 151-742, Korea

E-mail address: sheen@math.snu.ac.kr

School of Mathematics, University of New South Wales, Sydney 2052, Australia

E-mail address: sloan@maths.unsw.edu.au

Department of Mathematics, Chalmers University of Technology, S-412 96 GöteBORG, SwEden

E-mail address: thomee@math.chalmers.se 